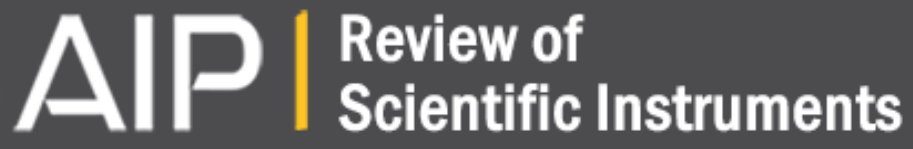

\section{A high precision, compact electromechanical ground rotation sensor}

V. Dergachev, R. DeSalvo, M. Asadoor, A. Bhawal, P. Gong, C. Kim, A. Lottarini, Y. Minenkov, C. Murphy, A. O'Toole, F. E. Peña Arellano, A. V. Rodionov, M. Shaner, and E. Sobacchi

Citation: Review of Scientific Instruments 85, 054502 (2014); doi: 10.1063/1.4875375

View online: http://dx.doi.org/10.1063/1.4875375

View Table of Contents: http://scitation.aip.org/content/aip/journal/rsi/85/5?ver=pdfcov

Published by the AIP Publishing

\section{Articles you may be interested in}

A high-precision mechanical absolute-rotation sensor

Rev. Sci. Instrum. 85, 015005 (2014); 10.1063/1.4862816

Effective excitation by single magnet in rotation sensor and domain wall displacement of FeCoV wire J. Appl. Phys. 109, $07 E 531$ (2011); 10.1063/1.3564948

Mechanical characterization of the Varian Exact-arm and R-arm support systems for eight aS500 electronic portal imaging devices

Med. Phys. 37, 1707 (2010); 10.1118/1.3368604

Compact, low-cost, and high-resolution interrogation unit for optical sensors

Appl. Phys. Lett. 89, 201113 (2006); 10.1063/1.2390650

Compact low-power high-sensitivity angle sensor

Rev. Sci. Instrum. 75, 988 (2004); 10.1063/1.1669120

\section{GRANVILLE-PHILLIPS ${ }^{\circledR} \quad$ Introducing the First}

Advanced Vacuum Measurement Solutions

Vacuum Gauges:

Convectron", Micro-Ion, Stabil-Ion*,

Cold Cathode

Cold Cathode Gauge

worthy of the

Mass Spectrometers:

Vacuum Quality Monitors

Granville-Phillips name!

-Unsurpassed Accuracy

-Predictive \& Easy Maintenance

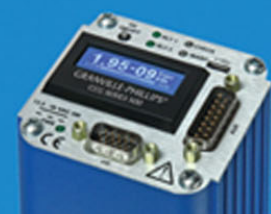




\title{
A high precision, compact electromechanical ground rotation sensor
}

\author{
V. Dergachev, ${ }^{1, a)}$ R. DeSalvo, ${ }^{1,2}$ M. Asadoor ${ }^{3,4}$ A. Bhawal, ${ }^{5,6}$ P. Gong, ${ }^{7,8}$ C. Kim, ${ }^{9}$ \\ A. Lottarini, ${ }^{10,11}$ Y. Minenkov, ${ }^{12}$ C. Murphy, ${ }^{13,14}$ A. O'Toole, ${ }^{15,16}$ F. E. Peña Arellano, ${ }^{17}$ \\ A. V. Rodionov, ${ }^{9,18}$ M. Shaner, ${ }^{3,19}$ and E. Sobacchi ${ }^{20}$ \\ ${ }^{1}$ LIGO Laboratory, California Institute of Technology, MS 100-36, Pasadena, California 91125, USA \\ ${ }^{2}$ University of Sannio, C.so Garibaldi 107, Benevento 82100, Italy \\ ${ }^{3}$ Mayfield Senior School, 500 Bellefontaine Street, Pasadena, California 91105, USA \\ ${ }^{4}$ Oklahoma State University, 219 Student Union, Stillwater, Oklahoma 74074, USA \\ ${ }^{5}$ Arcadia High School, 180 Campus Drive, Arcadia, California 91007, USA \\ ${ }^{6}$ Carnegie Mellon University, 5000 Forbes Ave, Pittsburgh, Pennsylvania 15213, USA \\ ${ }^{7}$ Department of Precision Instrument, Tsinghua University, Beijing 100084, China \\ ${ }^{8}$ School of Industrial and System Engineering, Georgia Institute of Technology, Atlanta, \\ Georgia 30332-0205, USA \\ ${ }^{9}$ California Institute of Technology, Pasadena, California 91125, USA \\ ${ }^{10}$ Department of Computer Science, University of Pisa, Largo B. Pontecorvo 3, 56127 Pisa, Italy \\ ${ }^{11}$ Department of Computer Science, Columbia University, 1214 Amsterdam Avenue, New York, \\ New York 10027, USA \\ ${ }^{12}$ Sezione INFN Tor Vergata, via della Ricerca Scientifica 1, 00133 Roma, Italy \\ ${ }^{13}$ School of Physics, The University of Western Australia, 35 Stirling Highway, Crawley, Perth, \\ Western Australia 6009, Australia \\ ${ }^{14}$ University of Melbourne Grattan Street, Parkville VIC 3010, Australia \\ ${ }^{15}$ University of California, Los Angeles, 405 Hilgard Ave, Los Angeles, California 90095, USA \\ ${ }^{16}$ Michigan Technological University, 1400 Townsend Dr, Houghton, Michigan 49931, USA \\ ${ }^{17}$ National Astronomical Observatory of Japan, 2-21-1 Osawa, Mitaka, Tokyo 181-8588, Japan \\ ${ }^{18}$ Department of Electrical Engineering, University of California, Riverside, California 92521, USA \\ ${ }^{19}$ University of Edinburgh, Edinburgh EH9 3JZ, United Kingdom \\ ${ }^{20}$ Scuola Normale Superiore, Piazza dei Cavalieri 7, 56126 Pisa, Italy
}

(Received 11 December 2013; accepted 25 April 2014; published online 15 May 2014)

We present a mechanical rotation sensor consisting of a balance pivoting on a tungsten carbide knife edge. These sensors are important for precision seismic isolation systems, as employed in land-based gravitational wave interferometers and for the new field of rotational seismology. The position sensor used is an air-core linear variable differential transformer with a demonstrated noise floor of $1 \times 10^{-11} \mathrm{~m} / \sqrt{\mathrm{Hz}}$. We describe the instrument construction and demonstrate low noise operation with a noise floor upper bound of $5.7 \times 10^{-9} \mathrm{rad} / \sqrt{\mathrm{Hz}}$ at $10 \mathrm{mHz}$ and $6.4 \times 10^{-10} \mathrm{rad} / \sqrt{\mathrm{Hz}}$ at 0.1 $\mathrm{Hz}$. The performance of the knife edge hinge is compatible with a behaviorur free of noise from dislocation self-organized criticality. (C) 2014 AIP Publishing LLC. [http://dx.doi.org/10.1063/1.4875375]

\section{INTRODUCTION}

In this paper, we present a ground rotation sensortiltmeter-with a demonstrated noise floor upper limit (Figure 1) of $5.7 \times 10^{-9} \mathrm{rad} \mathrm{Hz}^{-1 / 2}$ at $0.01 \mathrm{~Hz}$ and $6.4 \times 10^{-10} \mathrm{rad} \mathrm{Hz}^{-1 / 2}$ at $0.1 \mathrm{~Hz}$. The instrument's position sensor demonstrated excellent performance at low frequencies, despite high seismic noise of Caltech laboratory. The experimental setup, in particular lack of identical sensors for comparison, was the main limiting factor of sensitivity measurements.

A number of experiments to measure tilt were previously described. ${ }^{1-7}$ The best low frequency result of $1 \mathrm{nrad}$ at $0.1 \mathrm{~Hz}$ was reported by Ref. 1. An upcoming paper ${ }^{8}$ reports an improved result above $0.1 \mathrm{~Hz}$.

The development of the tiltmeter was motivated by two long standing problems: unsatisfactory seismometer tilt sensitivity and nonlinear behaviour and related noise in flexures. The construction of the present instrument was intended

${ }^{a)}$ Electronic mail: volodya@caltech.edu as a prototype sensor for the seismic isolation systems of Advanced LIGO. ${ }^{9}$ The benefits of this improvement are described in paper. ${ }^{10}$

The initial tiltmeter design ${ }^{11}$ used linear variable differential transformer (LVDT) sensors as they were considered to be easier to implement, also developing in parallel a Michelson interferometer readout for higher resolution readout. ${ }^{12,13}$ As development progressed, it turned out that the initial LVDT sensitivity limitations were due to external electronics. The LVDT sensitivity was then improved to $10^{-11} \mathrm{~m} \mathrm{~Hz}^{-1 / 2}$ which was sufficient to meet the measurement goals of this detector.

The key technology of this tiltmeter is the replacement of metallic hinges with a knife edge hinge made of tungsten carbide. This material avoids the possibility of metal-specific $1 / f$ noise and provides for an ultra-thin flexure that is easy to fabricate.

This paper is organized as follows: first we give a brief overview of seismometers, flexures, and position sensors. This is followed by a general description of the mechanical design of the tiltmeter and of the experiment setup, with 

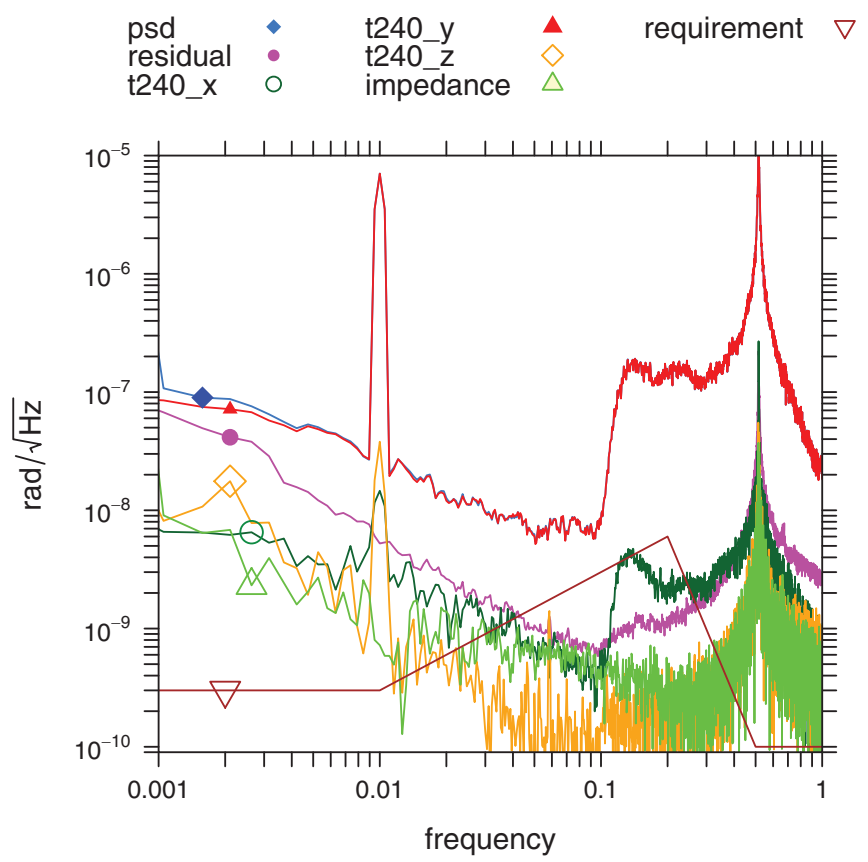

FIG. 1. Regression analysis of tiltmeter spectrum. The tiltmeter signal power spectral density (PSD) (blue trace) has been decomposed through coherence analysis (described in detail in Appendix A) into the sum of signals explained by four witness channels and the residual noise. The contributions of the $\mathrm{X}$, $\mathrm{Y}$, and $\mathrm{Z}$ channels of a Trillium 240 seismometer are marked with circle, filled triangle, and diamond correspondingly. The contributions show only the coherent part of the signal. The full seismometer PSD is shown on Figures 12 and 13. The residual noise channel describes the quadratic sum of the noise internal to the tiltmeter, the internal noise of the Trillium and the noise from mechanical connection between the two instruments. After taking into account statistical corrections the $95 \%$ confidence level upper limit on the tiltmeter noise is $5.7 \times 10^{-9} \mathrm{rad} / \sqrt{\mathrm{Hz}}$ at $10 \mathrm{mHz}$ and $6.4 \times 10^{-10} \mathrm{rad} / \sqrt{\mathrm{Hz}}$ at $0.1 \mathrm{~Hz}$. The larger residuals at low frequencies correspond to extra environmental noise seen by seismometer (Figure 13).

key details given on construction, sensors, and electronics. Next we discuss measurements. A thorough discussion of data analysis methods (in particular, the FFT-based regression) is found at the end of the paper.

\section{SEISMOMETERS}

A simplified schematic of traditional seismometer is shown in Figure 2. It consists of a mass $M$ which is connected by an ideal spring $k$ to the casing (deviations from elasticity are discussed in Sec. III). A viscous damping $c$ is assumed to exist, which can be either mechanical or electrical (via eddy current, for example).

This picture is very much idealized, for example, real springs are known to exhibit nonlinear behaviour which is discussed next. Also, while geophones or accelerometers are often constructed of only passive electro-mechanical parts as shown on the schematic, long-period seismometers usually incorporate some measure of electronic feedback.

An acceleration applied to the instrument casing along the measurement axis results in a force exerted by the spring on the mass $M$. Due to inertia, the mass will follow the applied force with a lag creating a temporary displacement from the equilibrium position. A seismometer measures this displacement with a suitable internal position sensor. Geophones

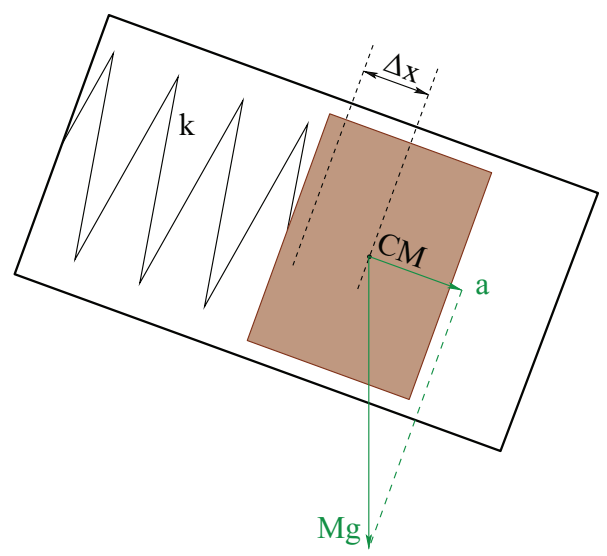

FIG. 2. A seismometer. Tilt of the case allows the projection $a$ of the acceleration of gravity $M g$ to act on the mass $M$ in a manner that, according to the principle of equivalence, is indistinguishable from horizontal acceleration. Here, $\Delta x$ is the displacement of the center of mass $C M$ from the equilibrium position, $k$ is the spring constant.

commonly employ a velocity readout, such as provided by a coil moving relative to a magnet. An accelerometer can be constructed by either direct sensing of acceleration (achieved, for example, with a piezo element) or by a force-feedback approach.

The behaviour at high and low frequencies (relative to the natural frequency of the mass-spring oscillator) is qualitatively different.

At high frequencies the mass is effectively stationary and defines an inertial reference frame. The displacement measurement is thus the measurement of the actual movement of the ground (i.e., the instrument case) relative to that inertial frame.

At low frequencies-below the resonant frequency-the behaviour is quite different. Assuming the spring has Hooke's constant $k$, the internal displacement $\Delta x$ of the center of mass $C M$ is proportional to the acceleration of the instrument casing according to the formula $\Delta x=\frac{a}{M k}$. In particular, if an ideal instrument at equilibrium was moved to a new position, after the movement, it will settle to the same equilibrium point betraying no sign that it was moved.

The mass-spring system acts as a linear transducer from acceleration to internal displacement. If a record of the instrument output was made during movement one can reconstruct the overall displacement by twice integrating the acceleration data, a procedure that amplifies measurement errors at low frequencies. The stability of the spring constant $k$ directly impacts the measurement noise.

An additional problem arises in a horizontal accelerometer if the levelling of the instrument is altered-for example, by deformation of its base or from hinge hysteresis. The change in levelling will cause the gravitational force of Earth to acquire a non-zero projection on the instrument axis. As the force is constantly present, this results in a persistent internal displacement of the mass which the instrument reports as a continual acceleration along the measurement axis. A naive integration of such a result can be rather perplexing as the experimenter might conclude that the instrument is accelerating and is travelling a growing distance while attached to an immobile object. 
Of course, from the point of view of general relativity the reading is perfectly correct-we are simply observing a manifestation of the equivalence principle which maintains that the effect of gravitational acceleration is indistinguishable from the influence of an accelerated frame.

To solve the issue of confusion between tilt and acceleration we must make a measurement where the prerequisites of the equivalence principle do not apply. Our experimental setup evades the equivalence principle on two grounds:

- the very presence of the gravitational force of generally constant amplitude is due to the support of the instrument (and the laboratory) by Earth which thus provides a system of reference independent from purely inertial frames of general relativity.

- the direction of the gravitational force is assumed to remain constant within the precision of the instrument. This essentially requires that the experimenter (or any object of a similar mass) does not run around within $1 \mathrm{~m}$ of the instrument. As such movement would also produce ground and air distortions that affect the instrument much more strongly we consider this a particularly mild condition.

The last assumption effectively declares that any measurement of apparent change in the direction of the gravitational vector relative to the inertial frame is due to the horizontal component induced by the acceleration of the instrument.

\section{NOISE IN FLEXURES}

In the preceding discussion we have assumed that our spring follows ideal Hooke's law $F=k \Delta x$. In real flexures or springs this is clearly not the case. The most obvious violation results from the presence of dissipative terms in the metal which is easily observed on a mass-spring system suspended in vacuum. In addition, the spring's material can exhibit hysteresis, or even spontaneous changes in equilibrium point. For example, equilibrium random walk was observed in Virgo's inverted pendulum. ${ }^{14}$ It is also possible that the equilibrium point will be restored with a delay (anelasticity ${ }^{15}$ ). In these cases the effect will be indistinguishable from tilt and the instrument will report a constant or anomalous acceleration.

A careful measurement will reveal that the nature of losses can have complicated causes.

For example, an unloaded spring will expand or contract with change of temperature according to its thermoelastic coefficient. A loaded spring will provide a temperature dependent force that will show up as drifts with changes in instrument temperature. Moreover, a contraction in the spring due to external force will raise its temperature slightly. If this heat was to diffuse away and the force was then removed the spring would expand and cool, achieving a slightly smaller temperature and different equilibrium position.

Another troublesome cause of nonlinearity is due to mechanical materials acquiring their rigidity from interaction of many small elements - such as molecules, atoms, or dislocations. The energy needed to break and/or relocate such bonds is usually quite small (chemical bonds have energies on the

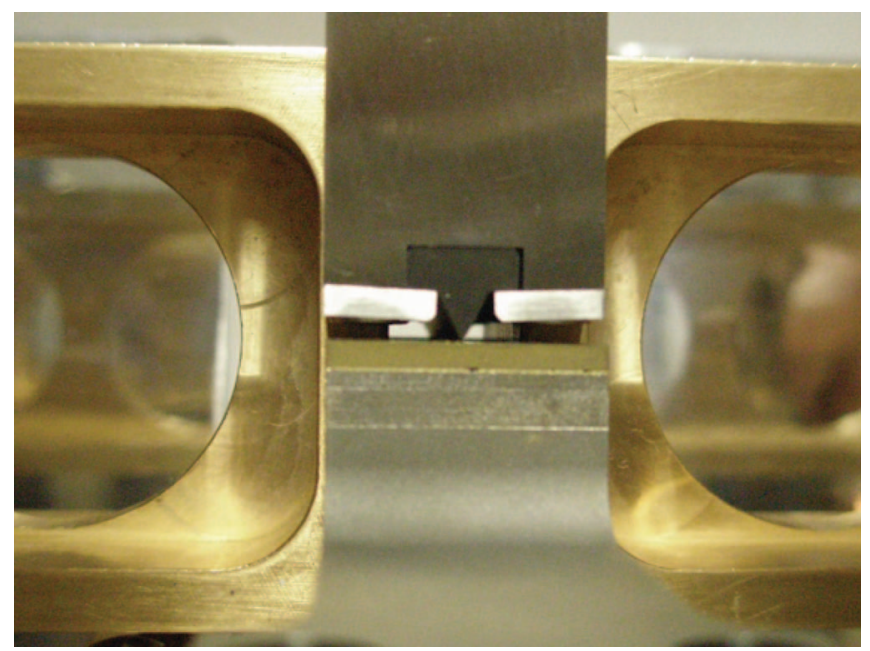

FIG. 3. Knife edge pivot is made of tungsten carbide grains held together by a cobalt binder.

scale of $1 \mathrm{eV}$ ) compared with the energy stored when the spring is compressed. For the spring to have ideal behaviour the stored mechanical energy needs to be evenly and stably distributed between constituent elements-as any variation of stress capable to break or relocate the bonds will lead to minute, permanent or metastable changes in spring length. This shows as measurement hysteresis-application and subsequent removal of external force does not return the spring to the original equilibrium point. ${ }^{16}$

In seismometers, it is desirable to employ a spring with a particularly low restoring force in order to achieve low resonant frequencies. In this instrument, we tried to build a hinge with zero elastic restoring force and reduced flexure hysteresis by eliminating the metallic flexures. To achieve this we designed the knife edge hinge shown in Figure 3 made of $0.8 \mu \mathrm{m}$ tungsten carbide powder bound with $8 \%$ cobalt. ${ }^{17}$

During initial studies of the tiltmeter using large scale deviations of an in-air instrument we observed no evidence of hysteresis above expected drifts from ground motion and electronics. ${ }^{18}$ The study of small deviation hysteresis was hampered by the inability to distinguish between tilt drift of the vacuum tank and instrument drift without a second identical witness instrument on the same platform.

As can be seen on the electron microscope image (Figure 4) the size of the surface features is on the order of few micrometers or less.

Consider tiltmeter angle excursions as large as $1 \times 10^{-5}$ $\mathrm{rad}$. For a wheel of less than $10 \mu \mathrm{m}$ diameter this corresponds to, at most, $0.2 \mathrm{~nm}$ travel, much smaller than grain size.

Thus, the pivoting knife edge does not actually roll on the anvil. A better model is the knife edge standing on a row of tungsten carbide grains, which bend slightly, forming an ultra-thin crystalline flexure.

\section{THE CHOICE OF THE POSITION SENSOR}

A tiltmeter readout requires a high precision position sensor. In this experiment, we employed a pair of aircore LVDT sensors which we found convenient to construct and 


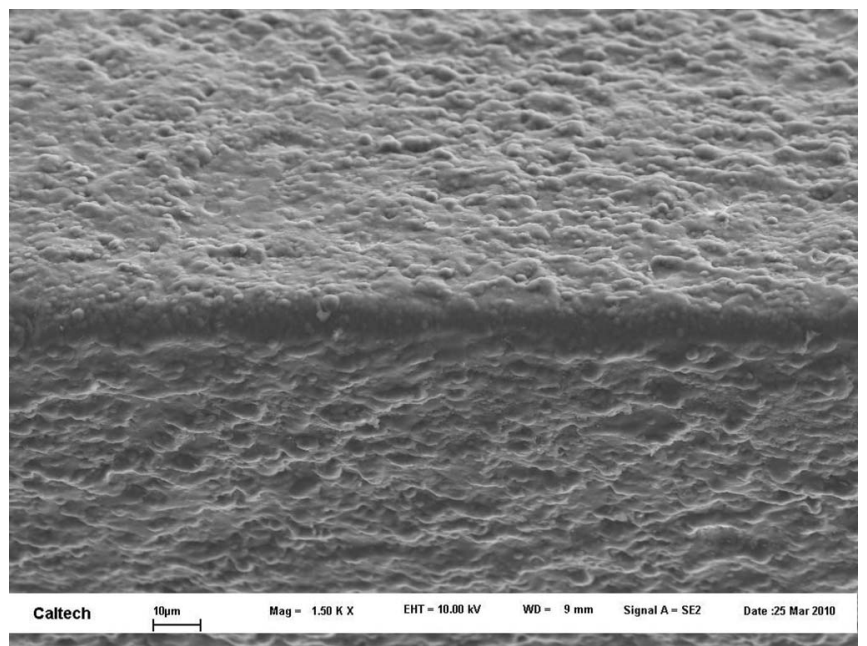

FIG. 4. Electron microscope photogram of knife edge. Typical size of surface feature is a few microns.

provided an easy path to achieve differential sensitivity of $10^{-11} \mathrm{~m} / \sqrt{\mathrm{Hz}}$ at low frequencies.

We briefly discuss this choice and compare it with two other position sensors: interferometric and capacitive.

At the moment the most accurate position measurements are attributed to interferometric sensors. Built on developments in lasers and optics over the past several decades, measurement accuracies down to $10^{-19} \mathrm{~m} / \sqrt{\mathrm{Hz}}$ have been demonstrated $^{19}$ at high frequencies $(100 \mathrm{~Hz})$.

An experimental setup to achieve such accuracy usually requires large resources and expertise. Moreover, the experience shows that the measurement is affected by strong $1 / f$ noise, unless carefully designed equal length arms and stabilized lasers are used. The survey of commercial interferometric sensors by the authors showed that while many could claim $1 \times 10^{-10} \mathrm{~m} / \sqrt{\mathrm{Hz}}$ above $100 \mathrm{~Hz}$, few could achieve $1 \times 10^{-9} \mathrm{~m} / \sqrt{\mathrm{Hz}}$ below $1 \mathrm{~Hz}$.

Making a custom interferometric readout is, of course, possible but difficult. A simple Michelson interferometer with an arm imbalance of only $300 \mu \mathrm{m}$ and a laser with a wavelength on the order of $1 \mu \mathrm{m}$ requires an absolute laser frequency stability better than $10 \mathrm{MHz}$ in order to avoid a measurement error of $10^{-11} \mathrm{~m}$. While lasers fitting this specification are available on the market their use is not very practical in this instrument. None of these issues pose a fundamental obstacle, ${ }^{20}$ as demonstrated by development of Laser Interferometer Space Antenna (LISA), ${ }^{21,22}$ but they would add considerable complexity and expense to the instrument. ${ }^{23}$

The stability of the Michelson readout is another issue the experimenter must be careful with. Most photodiodes would exhibit temperature-induced quantum efficiency drift on timescales of $\approx 100 \mathrm{~s}^{24,25}$ Only "photometric" diodes have this characteristic listed in the datasheet. ${ }^{26,27}$

Capacitive sensors are purely electronic devices just as LVDTs. There are three issues that an experimenter needs to consider carefully.

First, the position output of a simple capacitive sensor is usually quite nonlinear. This typically necessitates some form of electronic feedback on the mechanics to keep the sensor at its operating point. The performance of the instrument therefore relies also on the linearity and accuracy of actuation element. This can be challenging to achieve. For example, the actuation of a seismometer with a small electromagnet will require careful measurement of current at low frequency-a feat hard to achieve with off-the-shelf electronic components to accuracy better than $10 \mathrm{ppm}$ at $0.01 \mathrm{~Hz}$.

Second, the capacitive sensors depend on capacitance between two conductive surfaces. This can drift due to changes in material between the plates, or due to changes of the surface. ${ }^{28}$ For example, even pristine gold coatings were found to have considerable variations of surface potential. $^{29}$

Third, capacitive sensors rely on measuring an electric field between closely spaced electrodes. They tend to produce relatively strong, and strongly nonlinear, spurious forces that can pollute the actuation and the measurement.

An additional minor engineering challenge is the necessity of the two surfaces of a capacitive sensor to be in close proximity creating a large damping force when operating in air.

The LVDT sensors measure position by the differential inductance of three coils of a transformer. Commercial sensors usually have all coils wound on the static side of the instrument with a moving ferromagnetic core providing varying inductance. In addition, the instrument is shielded with ferromagnetic material to prevent external elements from influencing the magnetic field.

The use of ferromagnetic materials results in both sensing and actuation noise far above our requirement. For this reason we have chosen an air-core LVDT for our design, ${ }^{30} \mathrm{de}-$ spite the disadvantage of having to connect two signal wires to the moving part. We found it is very important to reduce leakage of the oscillating magnetic field outside the instrument, to minimize the residual forces that it can generate via Eddy current induction against the instrument case. The magnetic field leakage was nulled by adding two counterwound driving windings to the primary of the LVDT, thus nulling out most of the external field. Details of this geometry are given in Figures 8 and 9.

As the LVDT measures the mutual induction, the output voltage depends only on the current in the driving coil and on the mutual position of the coils. We obtained perfectly good results, presented in this article, from hand-wound coils on supports made of polyether ether ketone (PEEK). As the construction is fairly simple we were able to make multiple iterations of the design, altering the sensor geometry and density of coil windings to better match the requirements of the experimental setup.

Great care was taken to ensure that equal number of windings were wound on pairs and pairs of coils for best signal balancing and leakage force cancellation. Two thin, bare beryllium copper wires cross between the instrument case and the pivoting tiltmeter arm to feed the driving current to the primary. Beryllium copper was chosen for its good elastic properties. To minimize stray mechanical forces they are located as close as possible to the pivoting axis. We have also tried using unwound strands of litz wire, but saw increased noise level. 
Both primary and secondary coils were wound on cuplike supports, with the primary cup nested inside the secondary cup, with small clearance. The piston effect between the inner and outer cups would cause significant viscous damping in air. To avoid this, large diameter holes were opened on the sides of the cups, below the coils, as illustrated in Figure 9.

An important consideration in the construction of LVDT sensors is the quality and stability of the excitation current circuit, as any amplitude variation will appear as a proportional change of instrument reading. The usual remedy for this is to mount two LVDT sensors in opposite and use a ratiometric measurement. This scheme has the additional advantage of cancelling out drifts in the reference voltage of the data acquisition system. Thus, in effect, we employed four LVDTs, with two series connected opposing sensors on each side of the arm.

The influence of drifts in the reference voltage is reduced when the signal is sampled close to zero. It is thus very important to trim all sensors to operate near their null points.

High-quality DC-blocking capacitors proved necessary to impede near static currents in all windings of LVDT, but especially so in the driving coils.

The excitation current in our LVDTs was $\approx 30 \mathrm{~mA}$ and the excitation frequency was $6.1 \mathrm{kHz}$. The capacitance between the windings is of the order of $100 \mathrm{pF}$. Its variation can induce stray signals. Thus, it was very important to achieve a rigid mount not only in the translation direction along the sensor axis, but also perpendicularly.

As discussed later we achieved a sensitivity of $10^{-11} \mathrm{~m} \mathrm{~Hz}^{-1 / 2}$ demonstrating the suitability of the LVDT for precision position measurements.

\section{THEORY OF OPERATION}

The diagram of the experimental setup is shown in Figure 5. The brass balance arm is pivoting around the point $P$. There is a spring coupling $k$ and a damping coupling $c$ to the tiltmeter frame resting on the vacuum tank. The gravitational force $M g$ acts on the center of mass $C M$ of the tiltmeter.

Although the tiltmeter was designed with symmetric actuators below the arms for feedback operation, in this sensitivity measurement the actuators were removed.

The rest angle of the vacuum tank $\beta$ can be adjusted in a slow feedback loop with a thermal actuator to keep the LVDT sensors on the tiltmeter arm fully balanced during long data acquisition runs.

A Trillium 240 seismometer is placed at the top of the vacuum tank and is subject to the same thermal actuator tiltcorrection. All three analog channels $(\mathrm{X}, \mathrm{Y}, \mathrm{Z})$ report velocity and are acquired with a dedicated data acquisition module.

\section{A. Dynamics}

\section{Qualitative description}

Before giving an analytical description it is useful to consider high and low frequency limits of the tiltmeter dynamics.

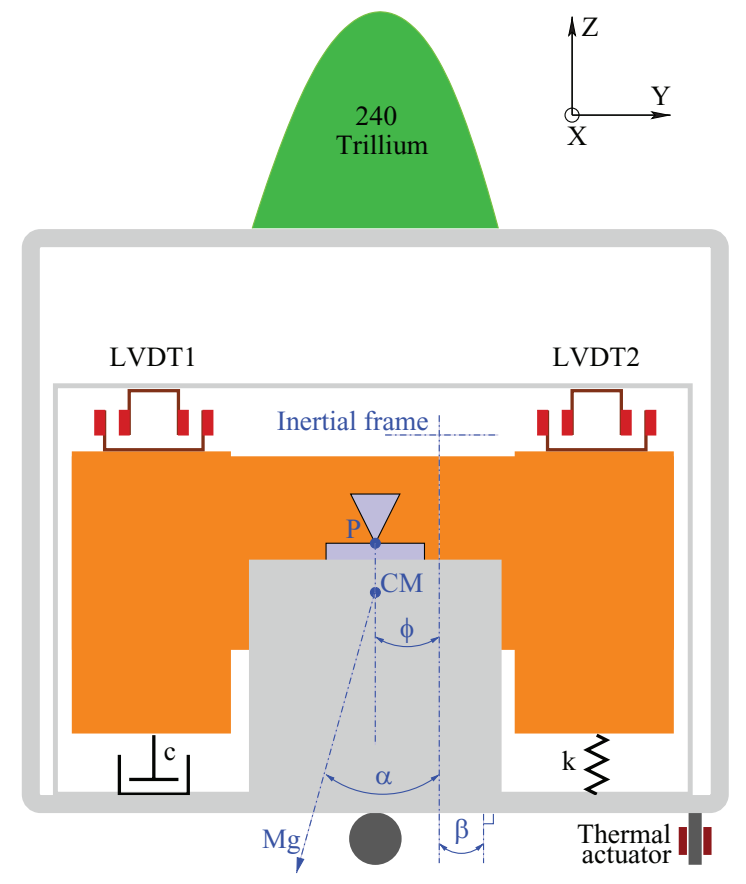

FIG. 5. A diagram of the tiltmeter in the vacuum chamber (not to scale). The Trillium 240 seismometer is mounted on top. This drawing focuses on the mechanical aspect of the experimental setup. Figure 10 describes electronics and data processing.

Like in a seismometer at frequencies high above the resonant frequency the balance beam has too much inertia to follow the excitation. Thus, the instrument output reflects the motion relative to the local inertial frame of the ground and the tiltmeter frame.

At low frequencies, and in the static regime the axis between the pivot point $P$ and the tiltmeter center of mass $C M$ simply follows the direction of the Earth gravitational force $M g$. The instrument, therefore, reads the angle between its frame and the gravitational field.

From the point of view of general relativity this frame is non-inertial-if the tiltmeter were in free fall its sensitivity at low frequencies would depend entirely on the spring constant and would be greatly reduced. Our measurement thus depends on the relation between the inertial frame of general relativity and the non-inertial frame provided by Earth.

The direction of the Earth's gravitational field is usually considered constant. However, the instrument sensitivity is such that this cannot be assumed for granted. For example, if $100 \mathrm{~kg}$ mass approaches the instrument from infinity to within $1 \mathrm{~m}$, the gravitational field would acquire a horizontal component of $6.8 \times 10^{-10} \mathrm{~g}$. This is within the sensitivity of the instrument provided the approach is rapid enough. Of course, in the practical implementation the effect of the floor bending under the weight will likely dominate the measurement.

A question might arise that if we allow for the change of the direction of the gravitational field, should we not also account for the change in the inertial frame as the space-time metric evolves? The answer is no. The components of the metric from the Earth's gravitational field are of the order $g / c^{2}=1.1 \times 10^{-16} \mathrm{~m}^{-1}$ and their contribution to the kinetic energy term can be safely ignored. 
Above resonance the tiltmeter is nearly immune to translation acceleration. Below resonance instrument acceleration adds to the gravitational acceleration vector.

The acceleration and rotation signals can be measured simultaneously by employing two integrated/colocated instruments with different resonant points. Giazotto ${ }^{6}$ describes other possible configurations.

\section{Analytic description}

To analytically describe the tiltmeter dynamics it is convenient to introduce three angle variables: $\alpha, \beta$, and $\phi$ defined in Figure 5.

The tiltmeter kinetic energy is given by $\frac{1}{2} I \dot{\phi}^{2}$ where $I$ is the moment of inertia of the arm and $\phi$ is the angle between the background inertial frame and the axis passing through the pivot point $P$ and the center of mass $C M$ of the balance beam.

The gravitational potential energy is given by $M g d \cos (\phi$ $-\alpha$ ) where $d$ is the distance between points $P$ and $C M$ and $\phi$ is the angle between the Earth gravitational force $M g$ and the background inertial frame.

The variable $\alpha$ describes the angle between the direction of Earth gravitational force and the axis passing through the pivot point $P$ and the center of mass of the balance beam $C M$. The angle $\beta$ describes the alignment of the vacuum tank and the tiltmeter frame to the Earth's gravitational force.

The sensors mounted on the ends of the balance beam thus measure the angle to the tiltmeter frame $\alpha-\beta$.

We describe the residual forces between the tiltmeter arm and the instrument case by the spring constant $k$ and the damping constant $c$. These forces are attributed mostly to the LVDT excitation currents, with small contributions from hookup wires and the knife edge.

The equation of motion of the tiltmeter is then

$$
I \ddot{\phi}+c(\dot{\phi}-\dot{\beta})+k(\phi-\beta)+M g d \sin (\phi-\alpha)=0 .
$$

Assuming that the angle $\phi-\alpha$ is small, we obtain

$$
I \ddot{\phi}+c \dot{\phi}+(k+M g d) \phi=c \dot{\beta}+k \beta+M g d \alpha .
$$

We measure the variable $\psi=\phi-\beta$ for which we compute the transfer function

$$
\psi(s)=\frac{-\left(I s^{2}+M g d\right) \beta(s)+M g d \alpha(s)}{I s^{2}+c s+k+M g d} .
$$

We find the natural frequency of the tiltmeter to be

$$
f_{0}=\frac{\omega_{0}}{2 \pi}=\frac{1}{2 \pi} \sqrt{\frac{k+M g d}{I}}
$$

and the quality factor as

$$
Q=c^{-1} \sqrt{I(k+M g d)} .
$$

After substitution we find

$$
\psi(s)=\frac{-\left(s^{2}+\frac{M g d}{I}\right) \beta(s)+\frac{M g d}{I} \alpha(s)}{s^{2}+s \frac{\omega_{0}}{Q}+\omega_{0}^{2}} .
$$

If $d=0$ we get $s^{2} \beta(s)$ in the numerator-which is the conventional transfer function for seismome-

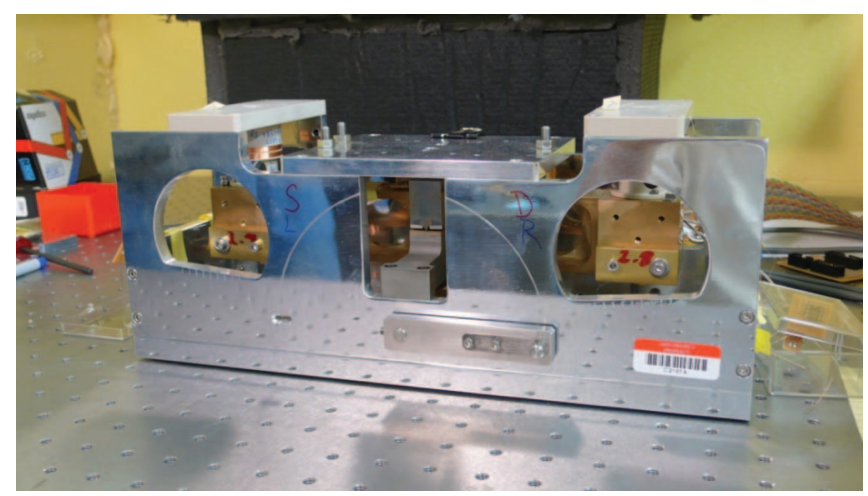

FIG. 6. A photograph of assembled tiltmeter. The length of the instrument is $38 \mathrm{~cm}$.

ters/accelerometers. Thus, the tiltmeter sensitivity at low frequencies is dominated by the gravitational spring.

We find it convenient to express the transfer function in terms of $\omega_{0}, Q$ and reduced spring constant $\omega_{k}^{2}=k / I$ :

$$
\psi(s)=\frac{-\left(s^{2}+\omega_{0}^{2}-\omega_{k}^{2}\right) \beta(s)+\left(\omega_{0}^{2}-\omega_{k}^{2}\right) \alpha(s)}{s^{2}+s \frac{\omega_{0}}{Q}+\omega_{0}^{2}} .
$$

\section{B. Thermal noise}

The thermal noise of a mechanical oscillator has been repeatedly considered in literature. ${ }^{9,31-35}$ Here, we present a concise computation for our case.

We neglect the thermal noise of the vacuum tank and the tiltmeter frame.

Using the fluctuation-dissipation theorem we find the noise power thermal density as

$$
S_{\phi}(\omega)=S_{\psi}(\omega)=\frac{4 k_{B} T \omega_{0} /(Q I)}{\left(\omega^{2}-\omega_{0}^{2}\right)^{2}+\omega^{2} \frac{\omega_{0}^{2}}{Q^{2}}} .
$$

\section{MECHANICAL CONSTRUCTION}

The assembled tiltmeter is shown on Figure 6. It consists of a brass balance beam (Figure 7) that provides leverage to the LVDT position sensors and contributes most of the inertial mass of the sensor. We summarized most important parameters in Table I. In the final version of the instrument the PEEK bridges and brass cubes shown in Figure 6 were replaced with aluminum parts.

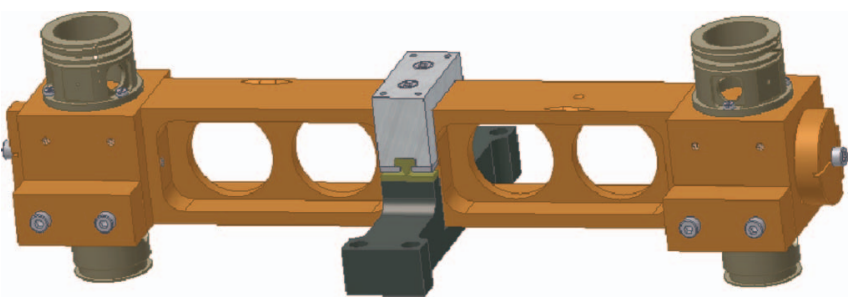

FIG. 7. CAD drawing of tiltmeter balance beam. The movable masses on the ends of arms are used to adjust center of mass with respect to the pivot point. 
TABLE I. Tiltmeter parameters. Mass and moment of inertia are only approximate as they do not take into account masses of LVDT coils and mounting hardware. The natural frequency and factor are given for two configurations described in the paper for the mechanical pendulum alone, without electronic feedback.

\begin{tabular}{ll}
\hline \hline Parameter & \multicolumn{1}{c}{ Value } \\
\hline Frame dimensions & $38 \mathrm{~cm} \times 14.7 \mathrm{~cm} \times 12 \mathrm{~cm}$ \\
Balance length & $\approx 30 \mathrm{~cm}$ \\
Mass & $\approx 4 \mathrm{~kg}$ \\
Moment of inertia (I) & $\approx 0.025 \mathrm{~kg} \mathrm{~m}^{2}$ \\
Balance materials & Brass, aluminum 6061, PEEK, \\
& copper wire \\
Knife edge material & Tungsten carbide \\
Knife edge length & $4.5 \mathrm{~cm}$ \\
Knife edge support points & $2 \mathrm{contacts} 7 \mathrm{~mm} \mathrm{long}$ \\
Natural frequency & $0.025 \mathrm{~Hz}, 0.511 \mathrm{~Hz}$ \\
Quality factor & 90,1400 \\
\hline
\end{tabular}

The beam is supported by the tungsten carbide knife edge resting on an anvil (Figure 3) manufactured from a tungsten carbide plate brazed on a steel support.

An aluminum frame supports the anvil and the static coils of the LVDT sensors. Aluminum was chosen as a frame material for its good rigidity, thermal conductivity, and absence of ferromagnetic properties.

Weights can be added to the ends of the beam balance to adjust the moment of inertia and position of the center of mass of the apparatus, thus tuning resonance frequency.

A lifting mechanism is used to separate the knife-edge from the anvil for transport and maintenance. When lifted the arm is positioned by a standard three point (cone, slot, flat) kinematic support with respect to the frame.

\section{LVDT SENSORS}

The two LVDTs mounted at the ends of the arm were initially based on design described in Ref. 30. Later this design was modified to reduce the force coupling from the primary coils on the arm to the frame. The new design is shown on the schematic in Figures 8 and 9. This configuration achieves
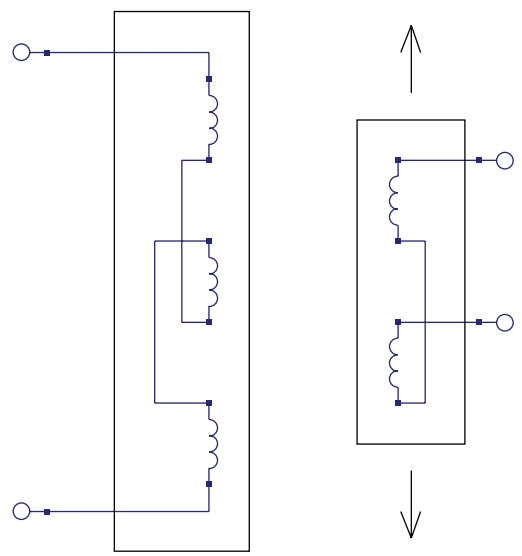

FIG. 8. Electromagnetic schematic of the LVDT sensor used in the tiltmeter. The right two coils form the secondary or receiver assembly that is mounted to the tiltmeter frame. The left three coils form the primary or the driver mounted on the balance beam.

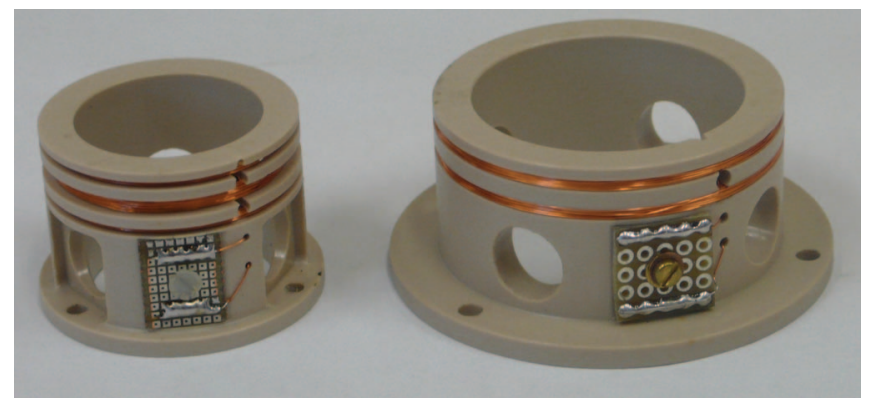

FIG. 9. Coiled LVDTs before installation. The three-coil driver is on the left. The number of windings in the first and third coil sum up to the same number of windings of the central coil, which is wound in the opposite direction. The two-coil receiver coil is on the right, the two coils have an identical number of windings, wound in opposite directions. The holes on the stand are to avoid piston-like damping for in-air operation.

greater confinement of the coil's magnetic fields at the expense of some loss of sensitivity.

The presence of two independent LVDT sensors allows to measure sensor self-noise by subtracting the outputs so that tilt signal cancels and only the common offset remains. The noise signal estimate obtained in this fashion includes noise contributions from analog electronics chain, data acquisition, mounting, and temperature variations. The spectrum of the common channel is shown in Figure 13 discussed in detail in Sec. X.

\section{ELECTRONICS}

The electronics subsystem of the experiment is shown in Figure 10. The "Spartan 3E-1600 Development Board" FPGA board made by Digilent, Inc serves as the main controller; providing clocks, acquiring the data from a Texas Instruments analog-to-digital converters (ADCs) ADS1278 and controlling output voltages via a Texas Instruments digital-to-analog converter (DAC) DAC8734.

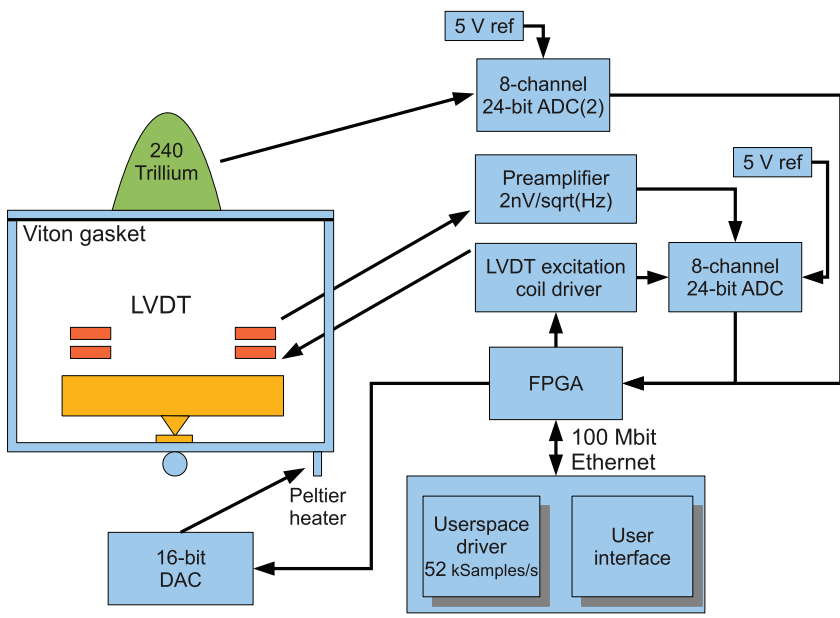

FIG. 10. Tiltmeter systems diagram. All data acquisition is synchronized to the same master clock. The signals from the Trillium 240 seismometer are sampled by an independent data acquisition board, with dedicated voltage reference. 
The master clock of the system is provided by an onboard $50 \mathrm{MHz}$ oscillator. We have also experimented using a more precise Wentzel oscillator but, as expected, this did not result in any improvement in performance. The $f_{0}=25 \mathrm{MHz}$ clock derived from the master clock is supplied to ADS1278 ADCs and the $f_{0} /(512 \times 8) \approx 6.1 \mathrm{kHz}$ clock is used for the LVDT excitation signal.

Eight data samples were acquired during each excitation cycle separated by $45^{\circ}$ phase relative to LVDT excitation. The board firmware was able to adjust the phase so that the first data acquisition cycle occurred at LVDT excitation phase of $22.5^{\circ}$ from signal peak.

The electronics were designed to minimize the effects of drift and $1 / f$ noise. The excitation current is produced by the coil driver which can drive up to $\approx 100 \mathrm{~mA}$ into the LVDT primary coils connected in series ( $200 \Omega$ total resistance). This assures that any fluctuations in excitation current or in excitation frequency are seen identically by both LVDT sensors.

The current generated by the LVDT driver had a symmetric triangular waveform which induced a square-wave voltage signal in the secondary. The slope of the driving waveform was determined by the voltage reference and integrator constant internal to the driver. The corners of the driving waveform had elevated noise level due to imperfections in the electronic switch. This noise was avoided by dropping every fourth sample from the acquired data.

The signals from $100 \Omega$ receiver coils pass through AD797 preamplifier and are sampled by a custom data acquisition system. ${ }^{36}$

The voltage reference on the ADC boards is only good to about $10 \mathrm{ppm} \mathrm{Hz}^{-1 / 2}$ at $0.01 \mathrm{~Hz}$ even when surrounded by foam insulation. In addition, the OPA1632 fully differential amplifiers present on the board are a significant source of heat which contributes to the thermally induced noise.

This precision in insufficient to accurately sample the tiltmeter output. To compensate for this and any changes in the drive current, a ratiometric measurement is made comparing the output of two LVDT sensors with the current supplied by the LVDT coil driver. Constant offsets larger than $10^{-5} \mathrm{rad}$ in measured signal should be avoided as they can directly couple reference noise into the measured output, limiting instrument sensitivity at low frequencies.

\section{ACTUATION AND FEEDBACK}

The instrument has been designed for feedback operation. For this different options were considered.

Figure 7 shows actuation coils mounted below the balance arm, which after assembly would face identical coils on the frame. The on-arm coils were wired in series to avoid stray torque from coupling with external fields, and the on-ground coils were wired with opposite sign to generate torque.

In this configuration actuation was intended by feeding the same AC signal both in the on-arm and in the on-frame coils, modulating the amplitude of one set.
In a different configuration the on-arm coils were replaced with small permanent magnets. Each end of the arm had two opposing magnets to reduce coupling with external fields.

In both cases the limiting problem was the lack of an instrument to determine the current (and force) produced by the actuators with the necessary precision $(1 \mathrm{ppm}$ at $0.01 \mathrm{~Hz})$. In the sensitivity characterization measurement the actuators were simply disconnected. To maintain the instrument's LVDTs near their null point, a slow feedback actuator was mounted below the vacuum tank housing the instrument, as shown in Figures 5 and 10.

\section{MEASUREMENTS}

Precision low frequency seismic measurements are delicate. Long data acquisition times are required in order to obtain the necessary statistics. This limits rather severely the number of experiment modifications that can be iterated to improve performance.

The presence of excess low frequency noise ( $1 / f$ or otherwise) in virtually all components can contaminate the data not only where actual noise is present but also at nearby frequencies (an example is discussed in Appendix B).

Comparison of seismic instruments requires very careful attention to how they are mounted on the common platform, insulated from temperature changes and air currents. Even the level of the seismic signal is important as the coherence between two instruments is known to degrade with larger levels of background seismic signal. ${ }^{37}$

Our experiment was located in the sub-basement level of West Bridge building in the Caltech campus. While this provided some protection from human induced seismic activity, it was far from perfect, especially during daytime, as can be seen in Figures 11, 15, and 16.

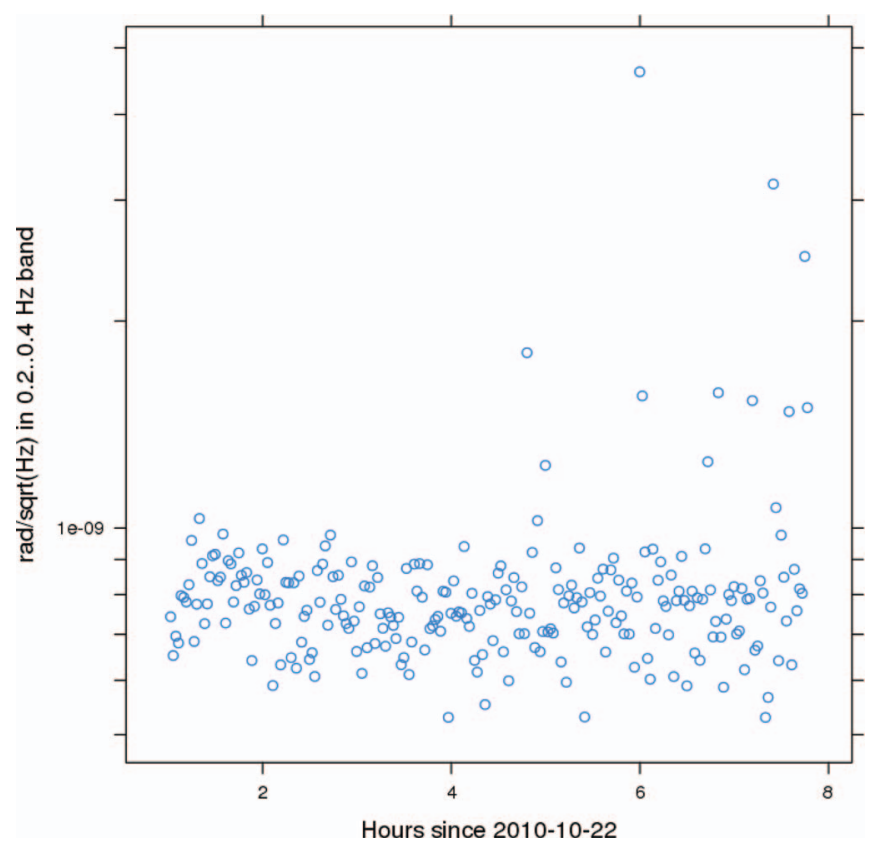

FIG. 11. Evolution of noise level in $0.2-0.4 \mathrm{~Hz}$ band. The high points after 5 a.m. correspond to early start of work activity nearby. 
In addition, a rather vigorous air exchange was maintained by the building ventilation system. This presented a problem both from the strong air currents and the air pressure fluctuations exerting a buoyancy force on the components of the experiment.

The standard way of characterizing an instrument is to place three (or at minimum two) of them side by side on the same rigid platform and separate the common signals (actual motion and tilt of the table) from the uncorrelated signals (internal noise from the individual sensors, independent environmental perturbations, etc.). This technique is commonly known as the huddle test.

Having just a single instrument, we characterized the tiltmeter by comparing its signal with a collocated Trillium 240 seismometer. The seismometer Y-signal is directly coupled through gravity and the vacuum tank to the tiltmeter signal. Because alignment is not perfect, the tiltmeter characterization is obtained extracting the tiltmeter signal fraction coherent with each of the three seismometer signals.

The tiltmeter and seismometer analog signals were sampled with different ADC boards, each calibrated with a dedicated $5 \mathrm{~V}$ reference. Thus, any drifts in two reference voltages or in other ADC circuitry show up as an additional component of residual noise shown in Figure 1.

\section{A. Operating environment}

Our vacuum tank was separated from the seismometersupporting top cover by a compressed viton gasket, which is not a high mechanical quality material and could not be expected to transmit faithfully horizontal accelerations as small as $10 \mathrm{~nm} / \mathrm{s}^{2}$.

Additional limitations were present. The vacuum tank was far above the pivot point of the steel hollow rod placed below the bottom (Figure 5). Thus, any horizontal forces (like air current pressure) generated extra tilt in the setup. The steel rod and the thermal actuator were situated on top of a steel plate which rested on the floor of the lab. The floor was covered with linoleum-a soft plastic material, which is easily deformed by applied forces and by any changes in temperature. All of these factors affect equally the tiltmeter and the seismometer, therefore they degrade the measurement of the seismic activity, but do not impair the evaluation of the residual signal of Figure 1 used to place an upper limit on instrument self-noise.

In order to provide the seismometer with a reduced noise environment and approach the low noise conditions described in Ref. 37 we stabilized the vacuum tank Y-direction tilt by feeding back the signal from the tiltmeter to the thermal actuator (bottom right of Figure 5). Only one degree of freedom was affected.

The reduction in background noise in the controlled direction can be seen in Figure 12. The $\mathrm{Y}$ axis of the seismometer that is aligned with the tiltmeter sensing axis is a factor of $\approx 2$ lower than the $\mathrm{X}$ axis data. Without actuation both curves would be at the same level. The steep $1 / f$ slope not present in

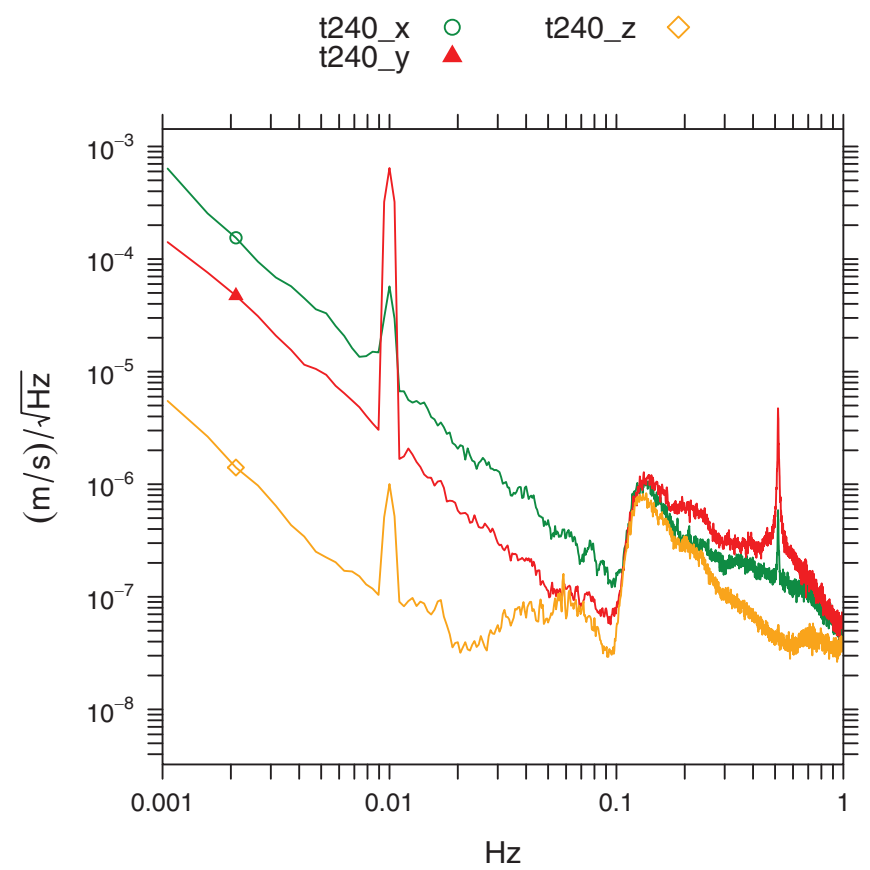

FIG. 12. Seismometer spectrum of the stabilized vacuum tank. The $\mathrm{Y}$ axis experiences a factor of $\approx 2$ reduction in noise relative to the $X$ axis. The sharp line at $0.01 \mathrm{~Hz}$ is a calibration line. The peak at $\approx 0.5 \mathrm{~Hz}$ is the eigenmode of the tiltmeter which leaks through the rolloff region (starting at $\approx 0.1 \mathrm{~Hz}$ ) of the feedback transfer function.

the output of the $\mathrm{Z}$ (vertical) axis is indicative of strong tilt signal in the laboratory.

In addition to the feedback signal a calibration line was injected at $0.01 \mathrm{~Hz}$.

\section{B. Performance, high frequency operation}

The tiltmeter performance is first illustrated on the spectrum plot (Figure 13). The tiltmeter signal (blue diamond) is seen overlaid with that of the Trillium $240 \mathrm{Y}$ axis (red triangle). The Trillium signal had been transformed into tilt units according to the analytical transfer function derived from geometrical measurements of the experimental setup. The LVDT calibration was done by means of a micrometer measuring large displacements. The two curves coincide over most of the spectrum.

The tiltmeter signal $\psi$ can be converted to the floor tilt by using the transfer function (7) with parameters $\omega_{0}=2 \pi$ $\times 0.511 \mathrm{~Hz}, Q=1400$ and $\omega_{k}^{2}=\frac{k}{I}=(2 \pi \times 0.826 \mathrm{~Hz})^{2}$. The large value of $\omega_{k}$ is due to forces from LVDT coils. The results, which assume that only tilt signal is present, are shown in Figure 14. This assumption is well warranted near $0.01 \mathrm{~Hz}$, but at frequencies above $0.1 \mathrm{~Hz}$ we expect a contribution of translational acceleration. A true simultaneous tilt and translational acceleration measurement would require two collocated balances.

The orange peak (marked with star) shows the expected contribution of the thermal noise of the tiltmeter. The brown curve (marked with triangle pointing down) shows the LIGO requirements from Ref. 10. 


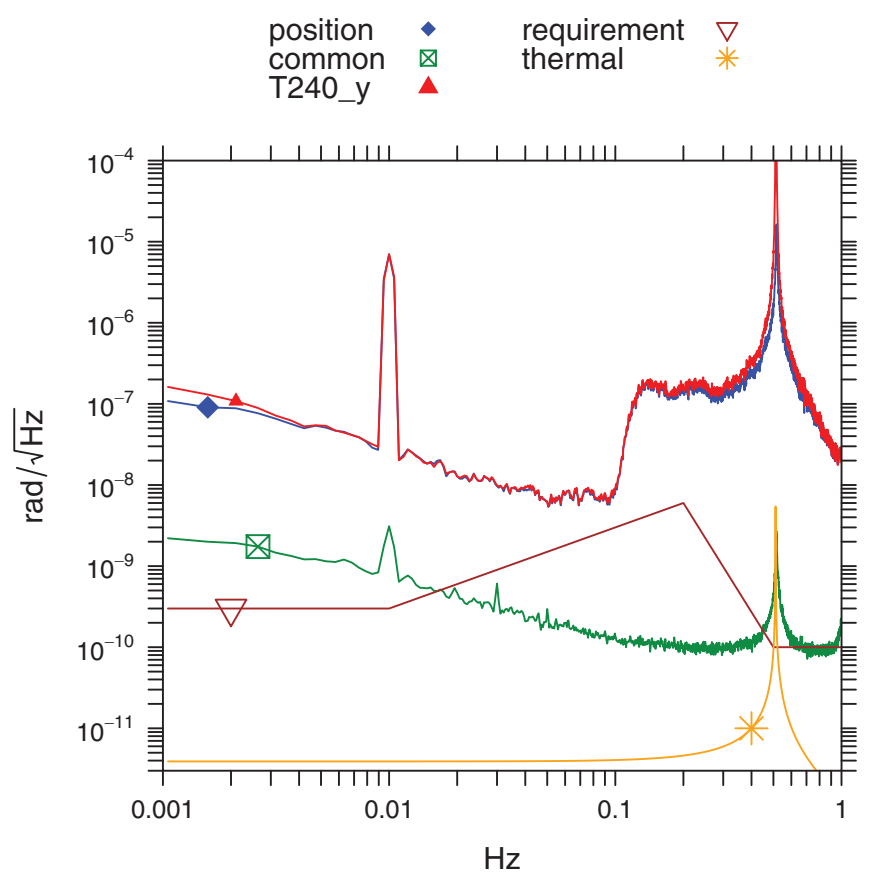

FIG. 13. Spectrum of the tiltmeter beam angle. Note the excess noise in the seismometer channel below $3 \mathrm{mHz}$ which corresponds to reduced coherence in Figure 1. The common signal is the average of two LVDT readouts, while the angular position is given by one-half of the difference.

The green curve (marked with a crossed square) shows the spectrum from the common mode of the tiltmeter sensors where tilt signal is cancelled out. The cancellation is not perfect; both the calibration line at $0.01 \mathrm{~Hz}$ and the proper mode of the tiltmeter leak through. The ratio of this leak through signal gives a good estimation of the cancellation accuracy,
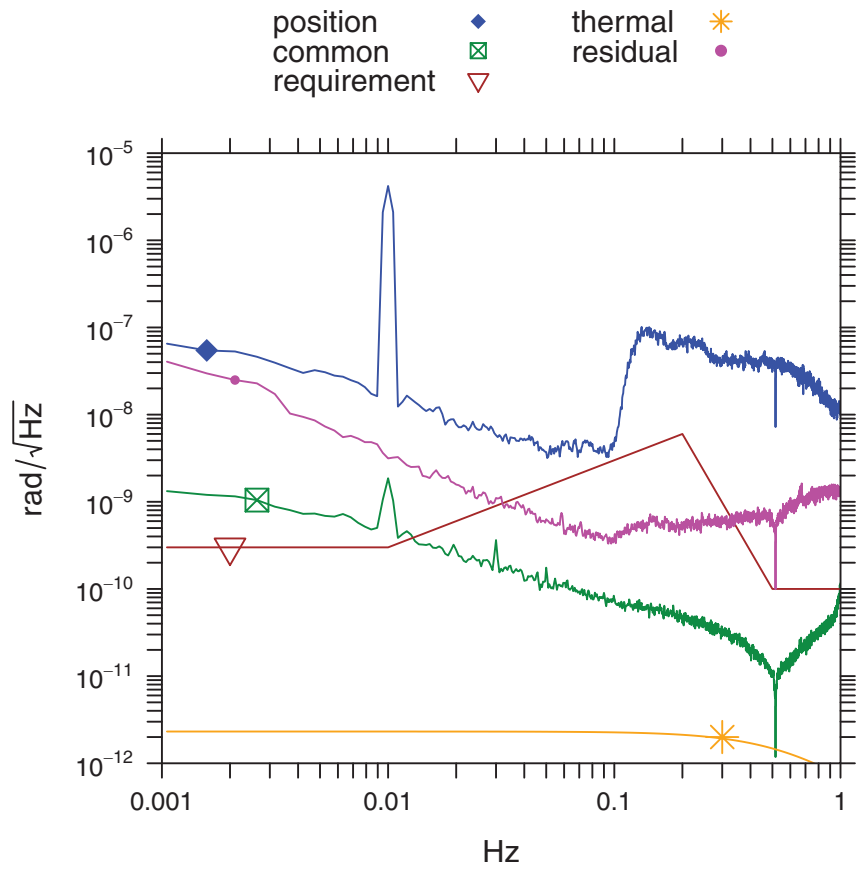

FIG. 14. Spectrum of floor tilt angle derived by applying the transfer function given by Eq. (7). The downward spike is due to imperfect modeling of the transfer function near the tiltmeter resonant frequency. i.e., 3 or 4 orders of magnitude. As the sensor data acquisition paths are independent and the data are combined digitally this curve shows that the LVDT readout is sufficiently sensitive to satisfy the requirements curve everywhere above 0.015 $\mathrm{Hz}$. The LVDT is located $0.125 \mathrm{~m}$ from the pivot point, thus LVDT sensitivity is better than $10^{-11} \mathrm{~m} \mathrm{~Hz}^{-1 / 2}$ for frequencies above $0.1 \mathrm{~Hz}$.

To probe the self-noise of the tiltmeter we employ the FFT regression described in detail in Appendix A. Briefly, it is equivalent to separating our signal into many narrow bands and then performing regression of data in each band onto witness signals. The result of the procedure is a separation of signals coherent between different measurement channels. The residual describes the incoherent noise.

The results are shown in Figure 1. The plot shows familiar curves of tiltmeter spectrum and the requirements curve alongside components of the tiltmeter signal that can be explained by the $\mathrm{Y}$ axis of the Trillium 240 signal, $\mathrm{X}$ and $\mathrm{Z}$ axes, and by an auxiliary signal measuring the impedance drift of the LVDT driver coil circuit.

The impedance signal is a useful witness signal because the LVDT driver was connected by rather long shielded twisted pair cables to the kovar wires on the flange of the vacuum tank which was in turn connected to the LVDT coils. There were several sources of drift stemming from change in the cable inductance from mechanical and thermal factors and changes in cable, LVDT and connection resistance.

In addition, a magenta curve (labelled with a filled circle) shows the residual signal that cannot be explained by any witness signals. It contains the sum of the tiltmeter selfnoise (both electrical and mechanical), the self-noise of the Trillium seismometer and any noise introduced by the imperfect attachment of instruments due to the vacuum tank or viton gasket, and any non-common environmental noise. With only two dissimilar instruments, it was not possible to further separate which part of the residual noise is contributed by the Trillium 240.

Taking into account statistical factors, we can be sure (with 95\% confidence level) that the tiltmeter beam angle self-noise is below $5.7 \times 10^{-9} \mathrm{rad} \mathrm{Hz}^{-1 / 2}$ at $0.01 \mathrm{~Hz}$ and $5.4 \times 10^{-10} \mathrm{rad} \mathrm{Hz}^{-1 / 2}$ at $0.1 \mathrm{~Hz}$.

\section{Low frequency operation}

By appropriately positioning weights the tiltmeter resonance frequency can be lowered. Figure 15 shows change in angle of the tiltmeter beam over one day. These data were taken with the tiltmeter configured to the resonance frequency of $25 \mathrm{mHz}$ and quality factor $Q=90$. The vacuum tank rested directly on the lab floor and no feedback control was applied.

We observe that daily variation in absolute tilt is $100 \mu \mathrm{rad}$. Even if we trimmed the instrument to have 0 tilt at one point in time after several hours the ground tilt will drift to a value that is six orders of magnitude above the target precision. To maintain accuracy either the sensor output needs to have absolute precision of $1 \mathrm{ppm}$ over this dynamic range, or, for instruments with electronic feedback, the applied force has to be measured with the same accuracy. 


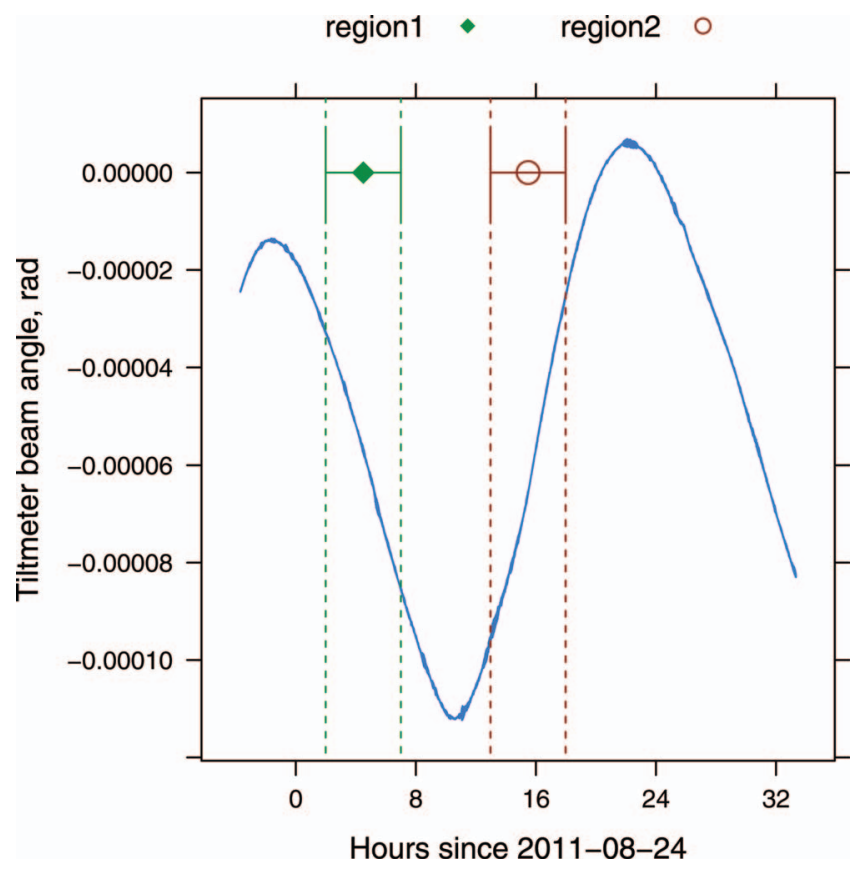

FIG. 15. Change in angle of the tiltmeter beam with time. The vacuum tank containing the tiltmeter was resting directly on the lab floor. No feedback was applied. The spectrum of data in regions 1 (night time) and 2 (day time) is shown in Figure 16.

Figure 16 shows two spectra obtained from data in regions 1 and 2 shown in Figure 15. During this particular day, the night time spectrum showed relatively small microseism. Day time data showed almost one order of magnitude larger noise.

One issue that arises in this configuration is that data near the resonance peak is subject to large phase shifts over a rather

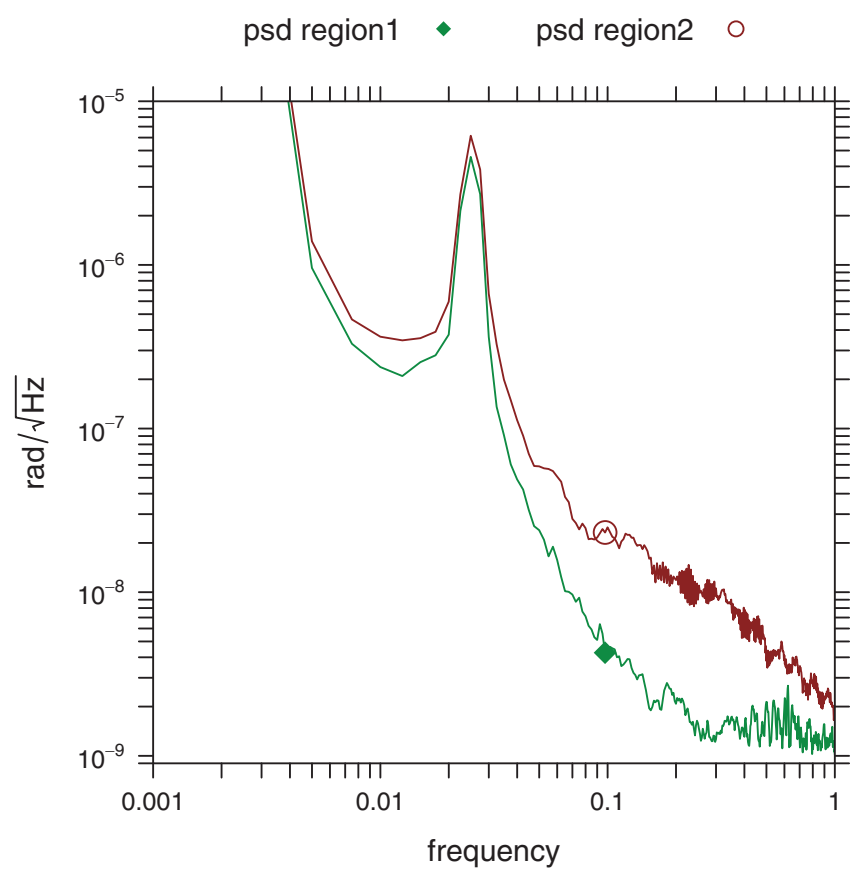

FIG. 16. Spectrum of the tiltmeter beam angle for regions 1 and 2 shown in Figure 15. The tiltmeter balancing weights were chosen to provide resonance frequency of $25 \mathrm{mHz}$. tiny (in absolute terms) region of frequency space. This makes it hard to convert beam tilt to floor tilt. One way to avoid this issue is to lower the resonance frequency. For example, in order to successfully collect precision data at $10 \mathrm{mHz}$ the resonance frequency has to be in the vicinity of $1 \mathrm{mHz}$.

We only found this practical with an electronic feedback loop, which could not match the sensitivity of an open loop sensor, as our instrumentation could only control applied force with $20 \mathrm{ppm}$ accuracy at $0.01 \mathrm{~Hz}$.

\section{CONCLUSIONS}

We have described construction and testing of a compact sensitive instrument capable of measuring tilts down to $5.7 \times 10^{-9} \mathrm{rad} / \sqrt{\mathrm{Hz}}$ at $10 \mathrm{mHz}$ and $6.4 \times 10^{-10} \mathrm{rad} / \sqrt{\mathrm{Hz}}$ at $0.1 \mathrm{~Hz}$ which, to our knowledge, exceeds the performance of presently available tilt sensors. The doubly differential LVDT sensors employed in the instrument have achieved noise floor of $1 \times 10^{-11} \mathrm{~m} / \sqrt{\mathrm{Hz}}$. The new knife edge flexure used in this tiltmeter performed very well showing no sign of excess noise, or of limiting the instrument performance.

\section{ACKNOWLEDGMENTS}

The instrument mechanics was manufactured by Galli \& Morelli, Acquacalda, Lucca, Italy on mechanical design produced by Mr. Gianni Gennaro, San Giuliano Terme Pisa.

This work has been done by members of LIGO Laboratory in California Institute of Technology, supported by funding from United States National Science Foundation. LIGO was constructed by the California Institute of Technology and Massachusetts Institute of Technology with funding from the National Science Foundation and operates under cooperative agreement PHY-0757058.

We have greatly benefited from comments and advice by Frank Seifert, Eric Gustafson, Virginio Sunnibale, and Randall D. Peters. We would also like to thank Brian Lantz, Fabrice Matichard, and Richard Mittleman.

We are very thankful to Takanori Sekiguchi for comments and suggestions for the early draft of this paper.

The work of E. Sobacchi was supported by INFN, Italy, in the framework of a student exchange program with NSF.

This document has LIGO Laboratory document number LIGO-P1300160-v3.

\section{APPENDIX A: PRACTICAL COMPUTATION OF AMPLITUDE SPECTRAL DENSITY AND FFT REGRESSION}

For completeness, we briefly review the method of obtaining the amplitude spectral density (used to produce Figure 13) and the FFT regression (Figure 1).

The basic tool employed is the computation of the time-frequency decomposition matrix of the signal, which schematic representation is shown in Figure 17.

The matrix can be computed for any real or complex time series $x_{t}$ by separating it into 50\%-overlapped segments of length $T$, applying Hann window to each segment and then performing independent Fourier transforms. This yields a ma- 


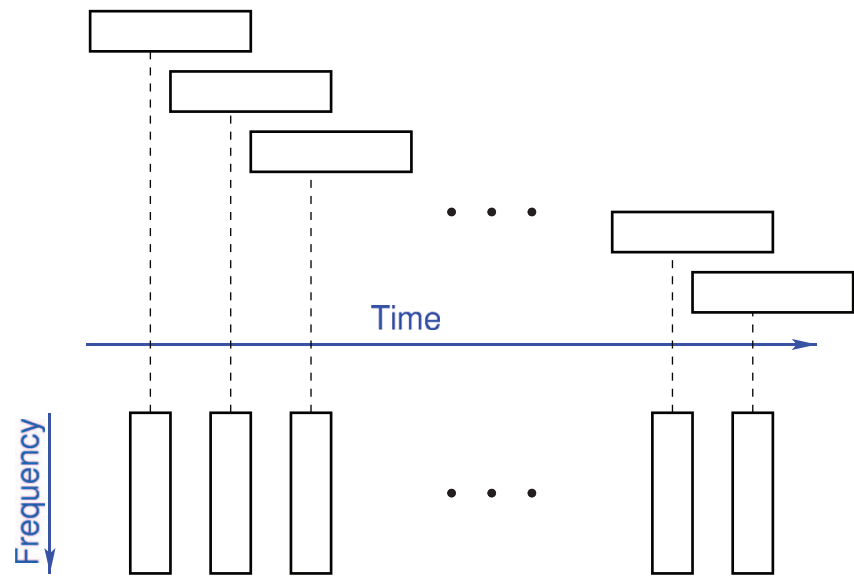

FIG. 17. FFT time-frequency matrix is constructed by separating data into small 50\%-overlapped data segments. Each data segment was Hann windowed and then was subjected to Fourier transform. The results of individual Fourier transforms are gathered in a matrix.

trix $Z[f, t]$ which rows are labeled by their FFT frequency bins and columns by the time of the beginning of the data segment used to compute the column:

$Z\left[f_{0}, t_{0}\right]=\int_{t_{0}}^{t_{0}+T} x_{t} e^{2 \pi i f_{0}\left(t-t_{0}\right)} \frac{1}{2}\left(1-\cos \left(2 \pi \frac{t-t_{0}}{T}\right)\right) d t$.

The power spectral density is then easily found as

$$
\operatorname{PSD}\left[f_{0}\right]=\frac{1}{T} \sum_{k=1}^{N}\left|Z\left[f_{0}, t_{k}\right]\right|^{2} .
$$

The power loss from windowing is minimized by using Welch's method of $50 \%$ overlapped intervals. We recommend that the reader be careful with Matlab's pwelch function. This function uses Hamming, not Hann window by default leading to larger leakage.

The amplitude spectral density is then computed as

$$
\operatorname{ASD}\left[f_{0}\right]=\sqrt{\operatorname{PSD}\left[f_{0}\right]}=\sqrt{\frac{1}{T} \sum_{k=1}^{N}\left|Z\left[f_{0}, t_{k}\right]\right|^{2}} .
$$

To compute the FFT regression we start with the main signal of interest $x_{t}$ and one or more witness signals $\left\{y_{t}^{i}\right\}_{i=1}^{K}$. For each of these signals we compute the time-frequency matrices $Z_{x}[f, t]$ and $Z_{y^{i}}[f, t]$.

Now we perform a linear regression separately on each row $f$ according to the model:

$$
Z_{x}\left[f_{0}, t\right]=\sum_{k=1}^{K} a_{k}\left[f_{0}\right] Z_{y^{k}}\left[f_{0}, t\right]+\operatorname{residual}\left[f_{0}\right] .
$$

The coefficients $a_{k}$ are complex and the absence of conjugate terms $\overline{Z_{y^{k}}\left[f_{0}, t\right]}$ is dictated by causality. The residual of the regression is frequency dependent and is a difference of the intrinsic noise of the instrument used to obtain $x_{t}$ and the linear combination of intrinsic noises of witness signals. Assuming a lack of correlation between the intrinsic noise of $x_{t}$ and witness signals the residual provides an upper limit estimate of the intrinsic noise of our instrument.
This effectively extends the usual linear regression of $x_{t}$ onto $y_{t}^{i}$ to the case when the regression coefficients are timeinvariant linear operators.

To create Figure 1 we plot the amplitude spectral density of the main signal, of the components of the regression decomposition $a_{k}\left[f_{0}\right] Z_{y^{k}}\left[f_{0}, t\right]$ and of the residual $\sqrt{\mid \text { residual }\left.\left[f_{0}\right]\right|^{2} / T}$.

For low frequency measurements the length $T$ is usually relatively large, which requires working with relatively small number of data segments $N$. For example, Figure 1 was made using $N=42$.

\section{APPENDIX B: A NOTE ON INFLUENCE OF DRIFTS IN THE COMPUTATION OF PSD}

Consider a signal drifting according to $s(t)=\alpha t$ over the entire measurement period. As our instrument has limited range, it is clearly unphysical to assume that the drift will continue indefinitely.

Instead, we might make a reasonable supposition that, perhaps, we are seeing a linear part of a sine wave $A \sin (2 \pi t / P)$ with a particularly long period $P$, such as shown in Figure 15. If the experimenter is only interested in a narrow band of frequencies away from zero it might be tempting to subtract this drift from the observed data as it corresponds to frequencies below the frequency of interest.

While drift-subtracted plots are useful during instrument development, in this paper all the plots were made on raw data, without subtracting drifts. Aside from general desire to be conservative, the determination that we see a long-period drift can only be done after a substantial amount of data has been collected and it would be challenging to filter out the drift in real time as it would be required for a control system.

Because the computation of the PSD involves stretches of data of length $T$ much smaller than $P$ the drift signal (or, in other words, strong very low frequency $1 / f$ noise) can leak into higher frequency bins. The amount of leakage depends strongly on the choice of window function used to compute the FFT.

Our choice of Hann window minimizes this issue, but does not eliminate it. One easily computes the amplitude spectral density of our signal as

$$
\operatorname{ASD}[f]=\frac{1}{4 \pi T^{3 / 2}} \frac{\alpha}{f(f-1 / T)(f+1 / T)} .
$$

As it can be expected, for a fixed frequency $f$ the drift contribution to amplitude spectral density decreases with larger FFT length $T$.

As is customary the plots shown in this paper have logarithmic frequency axis. On such a plot the left-most points are always just the first few bins of the Fourier transform. Converting into frequency bin units $f=k / T$ we find

$$
\operatorname{ASD}[f]=\frac{\sqrt{T}}{4 \pi} \frac{\alpha T}{k(k-1)(k+1)} .
$$

Thus, the researcher using a larger FFT length $T$ will see larger amplitude spectral density values in the lower frequency bins. Despite appearances this is not an indication of 
$1 / f^{3}$ noise, but simply the result of windowing drift signals of much longer duration.

To appreciate the influence of drifts, consider an example of a plot studying the behaviour near $f=0.01 \mathrm{~Hz}$ with FFT length $T=1000$ s. Then

$$
A(f)=\frac{\sqrt{T}}{4 \pi} \frac{\alpha T}{k(k-1)(k+1)}=0.0025 \mathrm{~Hz}^{-1 / 2} \cdot \Delta,
$$

where $\Delta=\alpha T$ is the total change across the interval $T$.

For contribution $A(f)<1 \times 10^{-10} \mathrm{~Hz}^{-1 / 2}$ we need $\Delta<4 \times 10^{-8}$. If, as an example, the source of the drift is a thermal expansion due to change in ambient temperature, then for thermal expansion coefficient of $1 \times 10^{-6} / \mathrm{K}$ (which is a value reached with invar, fused silica, zerodur, and other special materials, but for common materials is extraordinarily good), we need a thermal stability of better than $0.04 \mathrm{~K}$ over $1000 \mathrm{~s}$ interval.

Thus, control of drifts is crucial not only for final results, but also in everyday measurement as it allows for quicker experiments with smaller values of $T$.

${ }^{1}$ A. Takamori, A. Bertolini, R. DeSalvo, A. Araya, T. Kanazawa, and M. Shinohara, Meas. Sci. Technol. 22, 115901 (2011).

${ }^{2}$ A. N. Luiten, M. Beccaria, M. Bernadini, S. Braccini, C. Bradaschia, G. Cella, A. Ciampa, G. Curci, G. E. Cuoco, E. D’Ambrosio, V. Dattilo, R. DeSalvo, R. Del Fabbro, A. Di Virgilio, G. Feng, I. Ferrante, F. Fidecaro, A. Gaddi, A. Gennai, A. Giazotto, L. Holloway, P. La Penna, G. Losurdo, S. Malik, S. Mancini, H. B. Pan, F. Paoletti, A. Pasqualetti, D. Passuello, R. Poggiani, F. Raffaelli, A. Viceré, and Z. Zhang, Rev. Sci. Instrum. 68, 1889 (1997).

${ }^{3}$ Y. Cheng, J. Winterflood, L. Ju, and D. G. Blair, Classical Quantum Gravity 19, 1723 (2002).

${ }^{4}$ C. C. Speake and D. B. Newell, Rev. Sci. Instrum. 61(5), 1500 (1990).

${ }^{5}$ M. J. Usher and A. R. Mat Isa, Meas. Sci. Technol. 3, 574 (1992).

${ }^{6}$ A. Giazotto, Phys. Lett. A 376(5), 667 (2012).

${ }^{7}$ D. S. Durfee, Y. K. Shaham, and M. A. Kasevich, Phys. Rev. Lett. 97, 240801 (2006).

${ }^{8}$ K. Venkateswara, C. A. Hagedorn, M. D. Turner, T. Arp, and J. H. Gundlach, Rev. Sci. Instrum. 85, 015005 (2014)

${ }^{9}$ P. R. Saulson, Fundamentals of Interferometric Gravitational Wave Detectors (World Scientific, Singapore, 1994).

${ }^{10}$ B. Lantz et al., Bull. Seismol. Soc. Am. 99(2B), 980-989 (2009).

${ }^{11}$ A. O'Toole, F. E. Peña Arellano, A. V. Rodionov, M. Shaner, E. Sobacchi, V. Dergachev, R. DeSalvo, M. Asadoor, A. Bhawal, P. Gong, C. Kim, A.
Lottarini, Y. Minenkov, and C. Murphy, preprint, see http://dcc.ligo.org/ LIGO-P1400017.

${ }^{12}$ F. E. Peña Arellano, "Interferometric readout for tiltmeter," see https://dcc. ligo.org/P1300166.

${ }^{13}$ M. Shaner, F. E. Peña Arellano, E. Sobacchi, R. De Salvo, C. Kim, Y. Minenkov, C. Murphy, A. O’Toole, G. Pu, A. Rodionov, M. Asadoor, A. Bhawal, and V. Dergachev, "Interferometric readout for tiltmeter," see https://dcc.ligo.org/LIGO-G1001037.

${ }^{14} \mathrm{P}$. Ruggi, private communication (2013).

${ }^{15}$ C. C. Speake et al., Meas. Sci. Technol. 10, 430 (1999).

${ }^{16}$ R. DeSalvo, A. DiCintio, and M. Lundin, Eur. Phys. J. Plus 126, 75 (2011).

${ }^{17}$ P. I. O'Toole and T. S. Hudson, J. Phys. Chem. C 115(39), 19037 (2011).

${ }^{18}$ A. O’Toole, A. Bhawal, M. Asadoor, and R. DeSalvo, "Tiltmeter characterization," see https://dcc.ligo.org/LIGO-T1000340.

${ }^{19}$ B. Abbott et al. and The LIGO Scientific Collaboration, Nucl. Instrum. Methods Phys. Res., Sect. A 624, 223 (2010).

${ }^{20}$ F. E. Peña Arellano, H. Panjwani, L. Carbone, and C. C. Speake, Rev. Sci. Instrum. 84, 043101 (2013).

${ }^{21}$ M. Tröbs, P. Weßels, C. Fallnich, M. Bode, I. Freitag, S. Skorupka, G. Heinzel, and K. Danzmann, Classical Quantum Gravity 23, S151 (2006).

${ }^{22}$ P. McNamara, "Overview of LISA pathfinder (LISA-LPF-RP-0001)," see http://sci.esa.int/jump.cfm?oid=45822.

${ }^{23}$ G. de Vine, D. S. Rabeling, B. J. J. Slagmolen, T. T.-Y. Lam, S. Chua, D. M. Wuchenich, D. E. McClelland, and D. A. Shaddock, Opt. Express 17(2), 828 (2009).

${ }^{24}$ L. S. Lovinskii and V. I. Sorokin, Meas. Tech. 35(11), 1288 (1992).

${ }^{25}$ O. V. Moskovkin, Instrum. Exp. Tech. 44(1), 123 (2001).

${ }^{26}$ Hamamatsu, "Si photodiodes selection guide," see http://www.hamamatsu. com/resources/pdf/ssd/si_pd_kspd0001e.pdf.

${ }^{27}$ Hamamatsu, "Si PIN photodiode S5821 series datasheet," see http://www. hamamatsu.com/resources/pdf/ssd/s5821_series_kpin1010e02.pdf.

${ }^{28}$ C. C. Speake and C. Trenkel, Phys. Rev. Lett. 90, 160403 (2003).

${ }^{29}$ N. A. Robertson, J. R. Blackwood, S. Buchman, R. L. Byer, J. Camp, D. Gill, J. Hanson, S. Williams, and P. Zhou, Classical Quantum Gravity 23, 2665 (2006).

${ }^{30}$ H. Tariq, A. Takamori, F. Vetrano, Wang Chen Yang, A. Bertolini, G. Calamai, R. DeSalvo, A. Gennai, L. E. Holloway, G. Losurdo, S. Marka, M. Mazzoni, F. Paoletti, D. Passuello, V. Sannibale, and R. Stanga, Nucl. Instrum. Methods Phys. Res., Sect. A 489(1-3), 570-576 (2002).

${ }^{31}$ P. R. Saulson, Phys. Rev. D 42, 2437 (1990).

${ }^{32}$ S. K. Lamoreaux and W. T. Buttler, Phys. Rev. E: Stat., Nonlinear, Soft Matter Phys. 71(3 Pt 2A), 036109 (2005).

${ }^{33}$ E. Majorana and Y. Ogawa, Phys. Lett. A 233(3), 162 (1997).

${ }^{34}$ T. B. Gabrielson, IEEE Trans. Electron Devices 40(5), 903 (1993).

${ }^{35}$ S. Rowan, J. Hough, and D. R. M. Crooks, Phys. Lett. A 347, 25 (2005)

${ }^{36}$ More information is available at http://volodya-project.sourceforge.net/ daq.php.

${ }^{37}$ A. T. Ringler and C. R. Hutt, Seismol. Res. Lett. 81(6), 972 (2010). 